\title{
Grain Boundary Conductance Mechanisms of Ultra- fine Grained Ce02/BaCeO3 Based Electrolytes Fabricated by a Two-step Sintering Process
}

\section{Hao Ding}

Shandong University of Technology

\section{Tong Wu}

Shandong University of Technology

Haibin Sun ( $\square$ hbsun@sdut.edu.cn )

Shandong University of Technology https://orcid.org/0000-0002-8096-2968

\section{Shikai Zhao}

Shandong Industrial Ceramic Research \& Design Institute Co., Ltd

Jiao Li

Shandong University of Technology

Xue Guo

Shandong University of Technology

\section{Peng Wang}

Shandong University of Technology

\section{Research Article}

Keywords: Solid oxide fuel cells, Electrolytes, Grain boundary, $\mathrm{CeO} / \mathrm{BaCeO} 3$, Conductance mechanisms

Posted Date: November 5th, 2021

DOI: https://doi.org/10.21203/rs.3.rs-1012745/v1

License: @ (i) This work is licensed under a Creative Commons Attribution 4.0 International License.

Read Full License 


\title{
Grain boundary conductance mechanisms of ultra-fine grained $\mathrm{CeO}_{2} / \mathrm{BaCeO}_{3}$
} based electrolytes fabricated by a two-step sintering process

\author{
Hao DING, ${ }^{\text {a,b,1 }}$ Tong WU, ${ }^{\mathrm{a}, 1}$ Shikai ZHAO, ${ }^{\mathrm{c}}$ Haibin SUN, ${ }^{\mathrm{a}, ~ *}$ \\ Jiao LI, ${ }^{\mathrm{a}}$ Xue GUO, ${ }^{\mathrm{a}}$ Peng WANG, ${ }^{\mathrm{a}}$
}

a. School of Materials Science and Engineering, Shandong University of Technology, Zibo 255049,

China

b. Sinoma Advanced Nitride Ceramics CO., Ltd. Zibo 255000, China

c. Shandong Industrial Ceramic Research \& Design Institute Co., Ltd. Zibo 255000, China

\begin{abstract}
Aiming to clarify the grain boundary conductance mechanism of $\mathrm{CeO}_{2} / \mathrm{BaCeO}_{3}$ based electrolytes suitable for solid oxide fuel cells (SOFCs), Sm, Bi codoping $\mathrm{CeO}_{2} / \mathrm{BaCeO}_{3}$ (80 wt.\% $\mathrm{Ce}_{0.8} \mathrm{Sm}_{0.1} \mathrm{Bi}_{0.1} \mathrm{O}_{2-\delta}-20$ wt.\% $\mathrm{BaCe}_{0.8} \mathrm{Sm}_{0.1} \mathrm{Bi}_{0.1} \mathrm{O}_{3-\delta}$, BiSDC-BCSBi) electrolytes with ultra-fine grained $(110-220 \mathrm{~nm})$ and micron (1-1.8 $\mu \mathrm{m})$ structures were prepared by the two step sintering and conventional sintering method, respectively. Both electrolytes have pure phases corresponding to $\mathrm{CeO}_{2}$ and $\mathrm{BaCeO}_{3}$ without other purities. In the ultra-fine grained structure, apparent grain boundary conductivities measured at $350{ }^{\circ} \mathrm{C}$ and $400{ }^{\circ} \mathrm{C}$ are $1-2$ orders of magnitude higher than micron structures, thus resulting in dramatically enhanced electrical performances. This grain boundary effect can be attributed to two aspects. One is the decrease of space charge potential $\Delta \varphi(0)(0.165 \mathrm{~V}$ for ultrafine-fine grained ones, 0.396 $\mathrm{V}$ for micron ones). The other is the dilution of impurities (the impurity blocking term $\omega / d_{\mathrm{g}}$ is 0.94 for ultrafine-fine grained ones, and 0.53 for micron ones). In the ultra-fine grained electrolytes, no extra electronic conduction is introduced, and the ion migration number of $\mathrm{O}^{2-}$ is higher than that of $\mathrm{H}^{+}$. Finally, the ultra-fine grained BiSDC-BCSBi electrolytes maintain a good long-term stability in the operating condition of SOFCs at $600{ }^{\circ} \mathrm{C}$ for $100 \mathrm{~h}$.
\end{abstract}

Keywords: Solid oxide fuel cells, Electrolytes, Grain boundary, $\mathrm{CeO}_{2} / \mathrm{BaCeO}_{3}$,

\footnotetext{
${ }^{*}$ Corresponding author. 266 Xincun West Road, Zibo, 255000, PR China.

E-mail addresses: hbsun@sdut.edu.cn (H. Sun)

${ }^{1}$ These authors contribute equally to this work.
} 
Conductance mechanisms

\section{Introduction}

Solid oxide fuel cells (SOFCs) are regarded as one of the most promising energy conversion devices due to their advantages of high efficiency and environmental friendliness [1-5]. Aiming to avoid issues of cell packaging and electrode interface reactions caused by high operating temperatures, the current tendency of SOFCs is to decrease the operating temperature, but the consequent problem is deceased ionic conductivities of electrolytes [6]. Therefore, it is urgent to develop high conductive electrolytes suitable for intermediate-temperatures.

$\mathrm{CeO}_{2}$ and $\mathrm{BaCeO}_{3}$ based electrolytes have attracted much attentions due to their excellent oxygen ion conductivity and proton conductivity, respectively [7-9]. However, $\mathrm{Ce}^{4+}$ in $\mathrm{CeO}_{2}$-based electrolytes is easy to be partially reduced to $\mathrm{Ce}^{3+}$ in the reducing atmosphere, leading to undesirable electronic conductions [8]. And the poor chemical stability in $\mathrm{CO}_{2}$ and $\mathrm{H}_{2} \mathrm{O}$ hinders the application of $\mathrm{BaCeO}_{3}$-based electrolytes $[7,9]$. In recent years, composite electrolytes have been developed to suppress shortcomings of single electrolytes. Sun et. al reported that $\mathrm{CeO}_{2} / \mathrm{BaCeO}_{3}$ based composite electrolytes exhibited higher open circuit voltages (OCVs) than $\mathrm{CeO}_{2}$ based electrolytes, and better chemical stabilities against $\mathrm{CO}_{2}$ and $\mathrm{H}_{2} \mathrm{O}$ than $\mathrm{BaCeO}_{3}$ based electrolytes [10]. Based on this, in our previous works, enhanced electrical performances of $\mathrm{CeO}_{2} / \mathrm{BaCeO}_{3}$ based composite electrolytes were obtained by co-doping of $\mathrm{Sm}_{2} \mathrm{O}_{3}$ and $\mathrm{Bi}_{2} \mathrm{O}_{3}[11]$. The optimized total conductivity measured at $600{ }^{\circ} \mathrm{C}$ in wet air was $2.8 \times 10^{-}$ ${ }^{2} \mathrm{~S} \mathrm{~cm}^{-1}$, higher than the similar electrolytes (e. g, $\mathrm{Ce}_{0.85} \mathrm{Sm}_{0.15} \mathrm{O}_{1.92}-\mathrm{BaCe}_{0.83} \mathrm{Y}_{0.17} \mathrm{O}_{3-\delta}$, $1.70 \times 10^{-2} \mathrm{~S} \mathrm{~cm}^{-1}$ at $600{ }^{\circ} \mathrm{C}[12] . \mathrm{Ce}_{0.8} \mathrm{Sm}_{0.2} \mathrm{O}_{2-\delta}-\mathrm{BaCe}_{0.8} \mathrm{Sm}_{0.2} \mathrm{O}_{3-\delta}, 1.90 \times 10^{-3} \mathrm{~S} \mathrm{~cm}^{-1}$ at $600{ }^{\circ} \mathrm{C}[11]$.

The total conductivity of electrolytes consists of grain interior conductivity $\left(\sigma_{g i}\right)$ and grain boundary conductivity $\left(\sigma_{g b}\right)$. Generally, the apparent grain boundary conductivity is 100 to 1000 times lower than $\sigma_{g i}$, making it difficult to get ionic conductivity up a notch [13-15]. The grain boundary blocking effect may be described as two factors. One is the effect of space-charge layers [16]. The other is the enrichment of impurities on grain boundary regions [17]. 
Several researches confirm that the grain size has important effects on electrical properties for polycrystalline electrolytes [18-24]. For oxygen ion conductors, the commonly accepted view is that, the ionic conductivity can be dramatically enhanced with the decrease of grain sizes [25-27], which may be attributed to the promtion of ionic conductions due to the delocalized hopping, and the dilution of impurities along grain boundaries. But for Proton conductors, ones require larege-grained or grainboundary-free structures because grain boundaries own high barrier potential for proton conductions [28-30]. $\mathrm{CeO}_{2} / \mathrm{BaCeO}_{3}$-based electrolytes are mixtures of oxygen ion and proton conductors, the effect of grain sizes on electrical conductive behavior is still unclear.

Herein, micron and ultra-fine grained $\mathrm{CeO}_{2} / \mathrm{BaCeO}_{3}$-based electrolytes were fabricated, and carrier conduction behaviors effected by grain sizes were clarified. This work proved the promotion of ionic transportations benefiting from the decrease of grain sizes, which pointed in a direction for the development of such composite electrolytes.

\section{Experimental}

\subsection{Fabrication of electrolytes}

Based on our previous work [11], $\mathrm{Sm}_{2} \mathrm{O}_{3}$ and $\mathrm{Bi}_{2} \mathrm{O}_{3}$ co-doped $\mathrm{CeO}_{2} / \mathrm{BaCeO}_{3}$ based electrolytes (80 wt.\% $\mathrm{Ce}_{0.8} \mathrm{Sm}_{0.1} \mathrm{Bi}_{0.1} \mathrm{O}_{2-\delta}-20$ wt.\% $\mathrm{BaCe}_{0.8} \mathrm{Sm}_{0.1} \mathrm{Bi}_{0.1} \mathrm{O}_{3-\delta}, \mathrm{BiSDC}-$ $\mathrm{BCSBi}$ ) were taken as the research object due to their optimized conductivity. Micron and ultra-fine grained BiSDC-BCSBi electrolytes were fabricated by the conventional sintering (CS) and two-step sintering (TSS) process, respectively. For the CS process, the pellets were heated to $1250{ }^{\circ} \mathrm{C}$ at $3{ }^{\circ} \mathrm{C} \mathrm{min}^{-1}$ and kept for $3 \mathrm{~h}$, thus obtaining micron electrolytes. For the TSS process, the pellets were heated to $1100{ }^{\circ} \mathrm{C}$ at $3{ }^{\circ} \mathrm{C} \min ^{-1}$ and kept for $10 \mathrm{~min}$. Subsequently, the sintering temperature was rapidly lowered to 1050 ${ }^{\circ} \mathrm{C}$ and kept for $10 \mathrm{~h}$, thus achieving ultra-fine grained electrolytes.

\subsection{Characterizations}

Crystal structures were analyzed by the X-ray diffractometer (XRD, D8 Advance). Microstructures were observed by the scanning electron microscopy (SEM, Sirion 200). Oxidation states of electrolytes were analyzed by the X-ray photoelectron spectroscopy 
(XPS, Thermo Scientific ESCALAB 250XI XPS system). The electrochemical impedance spectroscopy (EIS) was tested using the electrochemical workstation (Zennium Pro) at the range from $0.1 \mathrm{~Hz}$ to $1 \mathrm{MHz}$ According to Eq. (1), the resistance value obtained from the EIS spectrum was converted to the conductivity $(\sigma)$.

$$
\sigma=\frac{L}{R S}
$$

Where $L$ and $S$ represent the thickness of electrolytes $(\mathrm{cm})$ and area $\left(\mathrm{cm}^{2}\right)$ of the electrolyte surface, respectively. $R$ represents the resistance value $(\Omega)$.

The blocking electrode method [31] was applied to confirm whether there is electronic conduction. The Keithley 2400 was used to test the $I-t$ curves at a polarization voltage of $0.8 \mathrm{~V}$. The electronic conductivity can be obtained from Eq. (2).

$$
\sigma_{e}(L)=\frac{L}{S} \times \frac{\partial I}{\partial E(L)}
$$

Where $I$ and $E$ are the steady-state current $(\mu \mathrm{A})$ and the polarization voltage $(\mathrm{V})$, respectively. $L, S$ are the same as in Eq. (1).

\subsection{Cell assembly and measurement}

Firstly, electrolyte pellets were ground to be a thickness of $0.4 \mathrm{~mm}$. And then, $75 \mathrm{wt} . \%$ $\mathrm{Ag}$ - 25 wt.\% $\mathrm{Ce}_{0.8} \mathrm{Sm}_{0.2} \mathrm{O}_{2-\delta}$ slurries were screen brushed on both sides of electrolytes as anode and cathode material. After being calcined at $880{ }^{\circ} \mathrm{C}$ for $2 \mathrm{~h}$, electrolytesupported single cells were assembled. The effective area of each single cell is $0.28 \mathrm{~cm}^{2}$.

The ionic transference number was obtained by the design of concentration cells. The details have been reported in our previous work [32]. For the measurement of oxygen ion transference number, the atmosphere on both sides of electrolytes are air and pure $\mathrm{O}_{2}$, respectively. For the measurement of proton transference number, the atmosphere on one side is pure $\mathrm{H}_{2}$, and the other side is the mixture of 97 vol.\% $\mathrm{Ar}$ and 3 vol.\% $\mathrm{H}_{2}$. The long - term stability of the single cell was measured at $600{ }^{\circ} \mathrm{C}$ for $100 \mathrm{~h}$, taking humid hydrogen (containing 3 vol.\% $\mathrm{H}_{2} \mathrm{O}, 40 \mathrm{~mL} \mathrm{~min}^{-1}$ ) and ambient air as fuel and oxidant, respectively.

\section{Results and discussion}

\subsection{Microstructure and phase composition}

Fig.1 (a) and (b) are the cross-sectional morphologies of the micron and ultra-fine 
grained BiSDC-BCSBi electrolytes, respectively. It can be seen that both electrolytes are in a dense state without obvious pores. The Nano Measurer software was used to carry out the grain size statistics. Grain sizes of micron electrolytes are roughly distributed in 1-1.8 $\mu \mathrm{m}$, while ultra-fine grained electrolytes are in 110-220 $\mathrm{nm}$. In the TSS process, the dominate grain growth mechanism is grain boundary diffusion instead of grain boundary migration while maintaining the escape of pores, thus achieving high grain boundary ratios.
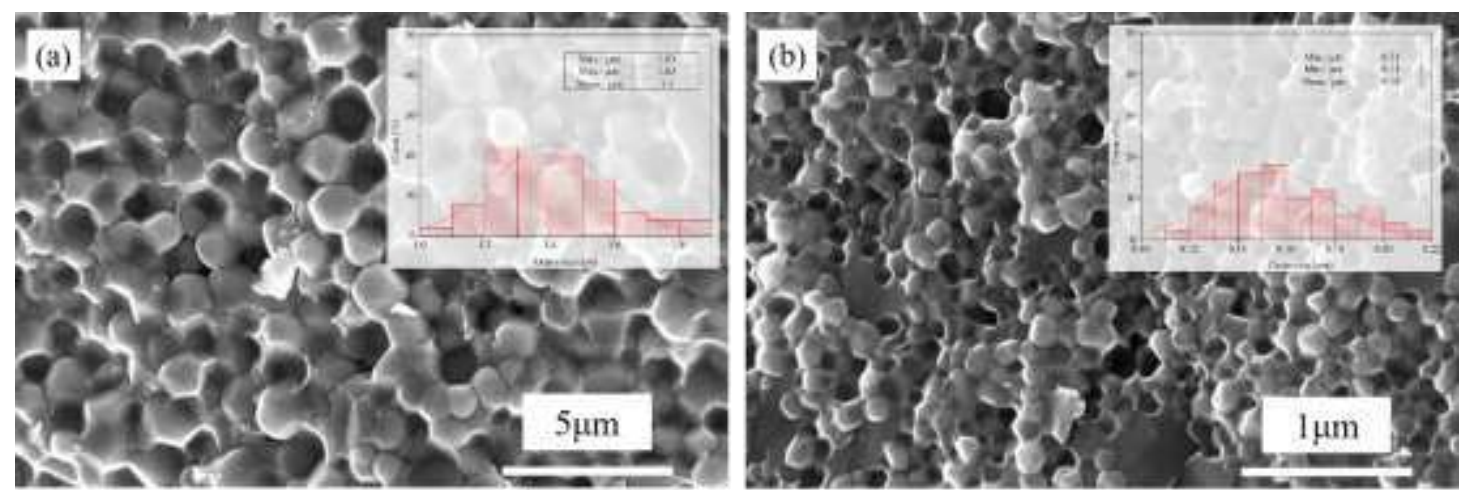

Fig. 1 SEM images of cross-sectional morphologies of (a) micron and (b) ultra-fine grained BiSDCBCSBi electrolytes.

Fig.2 shows the XRD spectra of micron and ultra-fine grained electrolytes. Compared with the standard cards of $\mathrm{CeO}_{2}$ and $\mathrm{BaCeO}_{3}$, it can be seen that both electrolytes are pure phases with only the diffraction peaks of $\mathrm{CeO}_{2}$ and $\mathrm{BaCeO}_{3}$, no impurity peaks or extra reactions are formed. The sintering process has no effect on the phase composition of electrolytes.

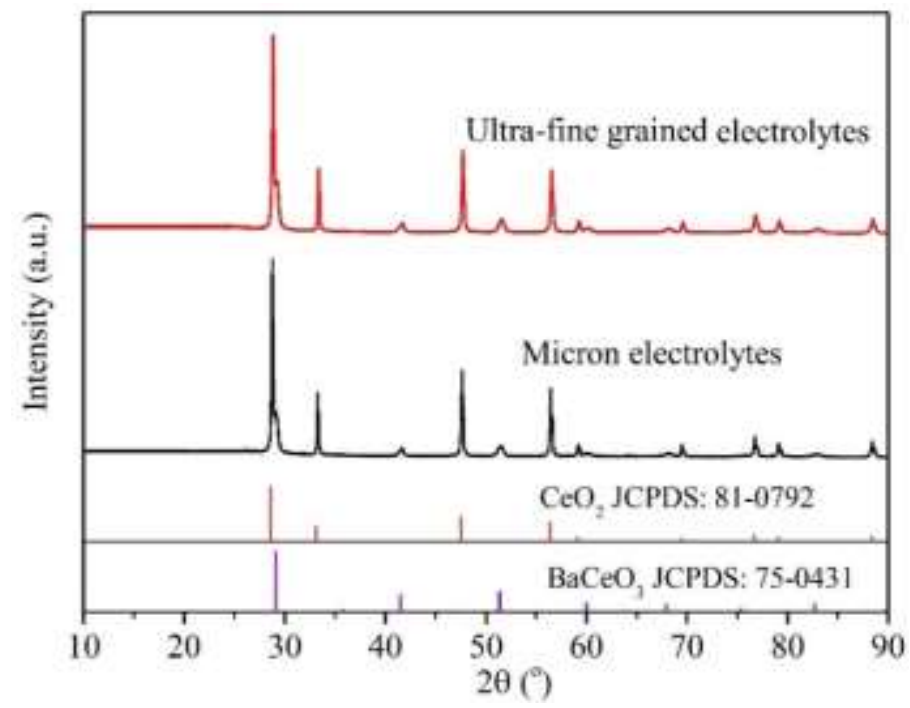

Fig. 2 XRD patterns of micron and ultra-fine grained BiSDC-BCSBi electrolytes. 


\subsection{Grain boundary conductance mechanism}

The EIS spectra of both electrolytes measured in humid air is shown in Fig. 3, in which two arcs can be clearly observed at testing temperatures of $350{ }^{\circ} \mathrm{C}$ and $400{ }^{\circ} \mathrm{C}$ There is an intersection with the real axis in the high frequency (HF) region, which corresponds to $R_{g i}$, and a semicircular arc in the shape of an oblate in the medium frequency (MF) and low frequency (LF) range. In order to distinguish $R_{\mathrm{gb}}$ from the semicircular arc, the Zahner Analysis software was used for circuit fitting. From the fitting data, characteristic capacitance values of $C_{1}$ and $C_{2}$ are calculated using Eq. (3), as listed in Table. 1 and Table. 2 [22].

$$
C_{i}=\frac{\left(R_{i} C P E_{i}\right)^{\frac{1}{n}}}{R_{i}}
$$

Where $R_{i}$ represents resistance values, and $C P E_{i}$ represents ideal capacitance values of $R_{i}$
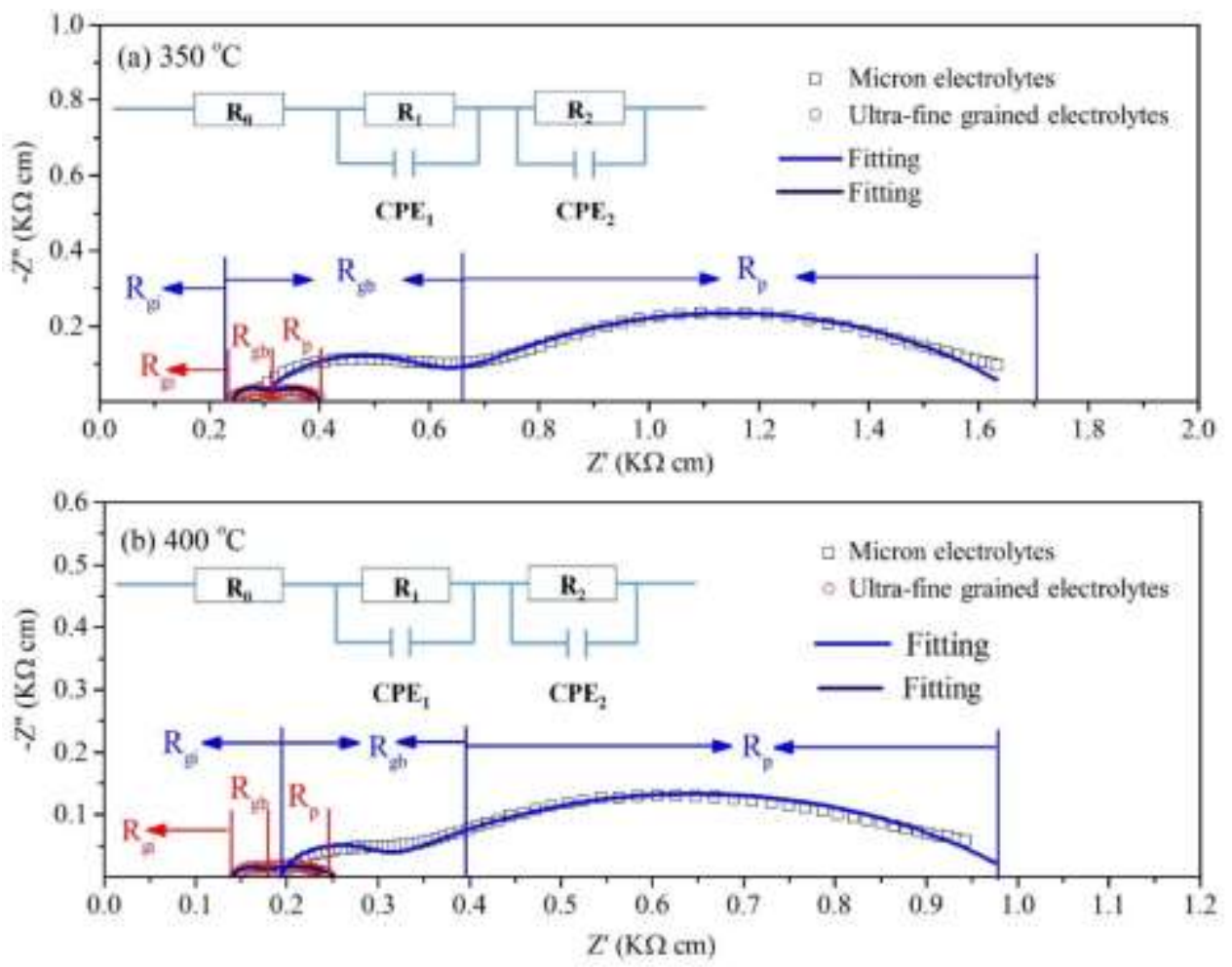

Fig. 3 EIS spectra and equivalent fitting circuits of micron and ultra-fine grained BiSDC-BCSBi electrolytes measured in humid air at (a) $350^{\circ} \mathrm{C}$, (b) $400^{\circ} \mathrm{C}$. 
Table 1 The fitting data and characteristic capacitance values of micron BiSDC-BCSBi electrolytes measured at different temperatures.

\begin{tabular}{cccccccccc}
\hline $\mathrm{T}\left({ }^{\circ} \mathrm{C}\right)$ & $R_{0}(\Omega)$ & $R_{1}(\Omega)$ & $C P E_{1}(\mathrm{~F})$ & $n_{1}$ & $C_{1}(\mathrm{~F})$ & $R_{2}(\Omega)$ & $C P E_{2}(\mathrm{~F})$ & $n_{2}$ & $C_{2}(\mathrm{~F})$ \\
\hline 350 & 14.23 & 19.43 & $2.17 \times 10^{-6}$ & 0.729 & $5.13 \times 10^{-8}$ & 47.14 & $0.59 \times 10^{-3}$ & 0.5 & $1.64 \times 10^{-5}$ \\
400 & 10.33 & 4.65 & $0.68 \times 10^{-6}$ & 0.985 & $5.61 \times 10^{-7}$ & 29.59 & $1.12 \times 10^{-3}$ & 0.5 & $3.71 \times 10^{-5}$ \\
\hline
\end{tabular}

Table 2 The fitting data and characteristic capacitance values of ultra-fine grained BiSDC-BCSBi electrolytes measured at different temperatures.

\begin{tabular}{cccccccccc}
\hline $\mathrm{T}\left({ }^{\circ} \mathrm{C}\right)$ & $R_{0}(\Omega)$ & $R_{1}(\Omega)$ & $C P E_{1}(\mathrm{~F})$ & $n_{1}$ & $C_{1}(\mathrm{~F})$ & $R_{2}(\Omega)$ & $C P E_{2}(\mathrm{~F})$ & $n_{2}$ & $C_{2}(\mathrm{~F})$ \\
\hline 350 & 12.91 & 3.95 & $1.84 \times 10^{-5}$ & 0.705 & $3.41 \times 10^{-7}$ & 4.88 & $2.05 \times 10^{-3}$ & 0.539 & $3.99 \times 10^{-5}$ \\
400 & 8.01 & 1.57 & $2.99 \times 10^{-6}$ & 1 & $2.99 \times 10^{-6}$ & 3.59 & $2.12 \times 10^{-3}$ & 0.538 & $5.21 \times 10^{-5}$ \\
\hline
\end{tabular}

It can be considered that $R_{0}$ is the $R_{\text {gi. }}$ Since $C_{1}$ is in the range of $10^{-6}-10^{-8} \mathrm{~F}$, it is judged that $C_{1}$ is the constant phase angle element of the grain boundary $\left(C P E_{\mathrm{gb}}\right)$ polarization process, thus $R_{1}$ represents $R_{\mathrm{gb}}$. Similarly, since $C_{2}$ is $10^{-5} \mathrm{~F}, C_{2}$ is considered as the constant phase angle element of the electrode polarization process $\left(C P E_{\mathrm{p}}\right)$, thus $R_{2}$ is the polarization resistance $\left(R_{\mathrm{p}}\right)$ [33-36]. Ultra-fine grained electrolytes have both lower $R_{\mathrm{gi}}\left(R_{0}\right)$ and $R_{\mathrm{gb}}\left(R_{1}\right)$ than micron ones, among which the decrease of $R_{\mathrm{gb}}$ is more significant. For example, $R_{\mathrm{gb}}$ of micron electrolytes is higher than $R_{\text {gi }}$ at $350{ }^{\circ} \mathrm{C}$, but $R_{g b}$ of ultra-fine grained electrolytes is just $30 \%$ of $R_{\text {gi }}$. According to Eq. (1), the grain-interior $\left(\sigma_{\mathrm{gi}}\right)$ and macroscopic grain boundary conductivity $\left(\sigma_{\mathrm{gb}}\right)$ of electrolytes can be obtained, as shown in Table. 3. Ultra-fine grained BiSDC-BCSBi electrolytes have slightly higher $\sigma_{\mathrm{gi}}$ and significant higher $\sigma_{\mathrm{gb}}$ than micron ones, thus obtaining higher total electrical conductivities.

The calculation formula for the apparent unit grain boundary conductivity $\left(\sigma_{g b}^{s p}\right)$ is shown in Eq. (4) to Eq. (6)

$$
\begin{gathered}
\sigma_{g b}^{s p}=\frac{\mathrm{L} \delta_{g b}}{S R_{g b} d_{g}} \\
\delta_{g b}=d_{g} \frac{C_{g i}}{C_{g b}} \\
\sigma_{g b}^{s p}=\sigma_{g b} \frac{C_{g i}}{C_{g b}}
\end{gathered}
$$

Where $\delta_{g b}$ is the thickness of a grain boundary layer, and $d_{g}$ is the average grain size. When the dielectric constant $\varepsilon_{g b}$ of the space charge layer is approximately equal to the 
dielectric constant $\varepsilon_{g i}, \delta_{g b}$ can be expressed as Eq. (5). Therefore, the calculation formula of $\sigma_{g b}^{s p}$ is converted into the Eq. (6) related to capacitance. It can be seen that the correlation between $\delta_{\mathrm{gb}}$ and $\sigma_{g b}^{s p}$ is relatively small. In order to facilitate calculation and comparison, $\delta_{g b}$ is taken as $5 \mathrm{~nm}[22,37] . \sigma_{g b}^{s p}$ values of micron and ultrafine grained electrolytes are listed in Table. 3. $\sigma_{g b}^{s p}$ of the ultrafine grained electrolytes is 1 to 2 orders of magnitude higher than that of micron electrolytes.

Table 3 Grain-interior, grain boundary, apparent unit grain boundary and total conductivities of micron and ultra-fine grained BiSDC-BCSBi electrolytes measured at different temperatures in humid air.

\begin{tabular}{clllll}
\hline Sample & $T\left({ }^{\circ} \mathrm{C}\right)$ & $\sigma_{\mathrm{gi}}\left(\mathrm{S} \mathrm{cm}^{-1}\right)$ & $\sigma_{\mathrm{gb}}\left(\mathrm{S} \mathrm{cm}^{-1}\right)$ & $\sigma_{g b}^{s p}\left(\mathrm{~S} \mathrm{~cm}^{-1}\right)$ & $\sigma_{t}\left(\mathrm{~S} \mathrm{~cm}^{-1}\right)$ \\
\hline Micron BiSDC- & 350 & $2.20 \times 10^{-3}$ & $1.79 \times 10^{-3}$ & $6.39 \times 10^{-6}$ & $0.98 \times 10^{-3}$ \\
BCSBi & 400 & $3.06 \times 10^{-3}$ & $6.89 \times 10^{-3}$ & $2.46 \times 10^{-5}$ & $2.12 \times 10^{-3}$ \\
Ultra-fine grained & 350 & $2.54 \times 10^{-3}$ & $8.48 \times 10^{-3}$ & $2.83 \times 10^{-4}$ & $1.99 \times 10^{-3}$ \\
BiSDC-BCSBi & 400 & $4.14 \times 10^{-3}$ & $2.02 \times 10^{-2}$ & $6.73 \times 10^{-4}$ & $3.43 \times 10^{-3}$ \\
\hline
\end{tabular}

The space charge layer and impurity blocking effects are usually used to explain the behavior of grain boundary conduction, which can be discussed use Eq. (7) [21].

$$
\frac{\sigma_{g b}^{s p}}{\sigma_{g i}}=\frac{\omega^{2}}{d_{g}^{2}} \frac{4 e \Delta \varphi(0)}{k_{B} T} \exp \left(-\frac{2 e \Delta \varphi(0)}{k_{B} T}\right)
$$

Where $k_{\mathrm{B}}$ is Boltzmann's constant, and $\omega^{2}$ is the direct contact area of grain to grain, $\Delta \varphi(0)$ is the space charge potential. Defined $\omega^{2} / d_{g}^{2}$ as the impurity blocking term. The higher the value, the weaker the influence of impurities such as $\mathrm{SiO}_{2}$ on the conduction path. Among them, impurities mainly come from the high-temperature volatilization of the furnace material, or from the preparation process. $\omega^{2} / d_{g}^{2}$ represents the clean part in the grain boundary region that is not covered by impurities. The value of $\omega^{2} / d_{g}^{2}$ is determined by the purity and grain size of the sample, and its value is 1 when there are no any impurities in the grain boundaries. The space charge potential can be calculated by Eq. (8) [17].

$$
\Delta \varphi(0)=-\frac{500 k_{B}}{e} \times k
$$

Where $k$ is the slope of the straight line drawn with $1000 / T$ as the abscissa and $\ln \left(T / \sigma_{g i}\right)$ 
as the ordinate, representing the chemical reaction rate constant.

Values of $k, \Delta \varphi(0)$ and $\omega / d_{g}$ are obtained from Eq. (7-8), and listed in Table. 4. It is obvious that the ultra-fine grained sample has lower space charge potential and higher $\omega / d_{g}$. That is, with the increase of grain boundary ratios, the ionic conduction is easier to get over barriers, and the impurity blocking effect is suppressed due to the impurity dilution at grain boundaries [21].

Table 4 Values of $k, \Delta \varphi(0)$ and $\omega / d_{\mathrm{g}}$ for micron and ultra-fine grained BiSDC-BCSBi electrolytes.

\begin{tabular}{ccc}
\hline Parameter & Micron BiSDC-BCSBi electrolytes & Ultra-fine grained BiSDC-BCSBi electrolytes \\
\hline$k$ & -9.184 & -3.815 \\
$\Delta \varphi(0)$ & 0.396 & 0.165 \\
$\omega / d_{\mathrm{g}}$ & 0.53 & 0.94 \\
\hline
\end{tabular}

In order to determine whether undesirable electronic conductions make contributions to the improved electrical conductivities of ultrafine grained BiSDC-BCSBi electrolytes, EIS testing on dry argon was performed to eliminate the influence of oxygen and water vapor in the air, thus inhibiting the conduction of $\mathrm{O}^{2}$ and $\mathrm{H}^{+}$. EIS spectra shown in Fig. 4 confirms that electronic conductance is present in both electrolytes.
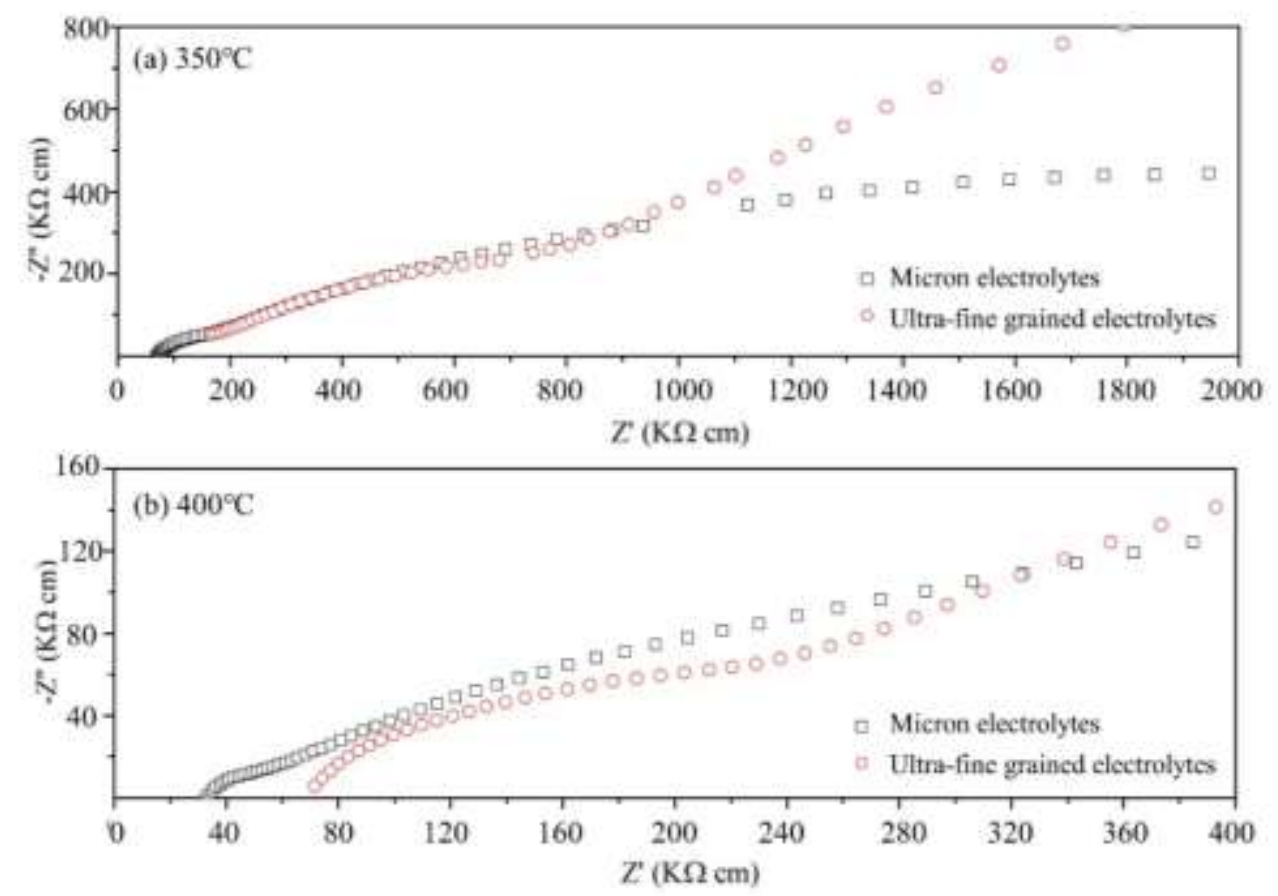

Fig. 4 Electrochemical impedance spectra of micron and ultra-fine grained BiSDC-BCSBi electrolytes at different temperatures in argon atmosphere. 
In order to accurately determine the electronic conductivity influenced by grain boundaries, the blocking electrode method was used to test the $I-t$ curves in air atmosphere through Keithley 2400 . Under the polarization voltage of $0.8 \mathrm{~V}$, the test results are shown in Fig. 5. According to Eq. (2), the electronic conductivities in air atmosphere are calculated and shown in Table. 5. For both micron and ultrafine grained BiSDC-BCSBi electrolytes, the electronic conductivity at $350-600{ }^{\circ} \mathrm{C}$ is roughly in the range of $10^{-12}-10^{-9} \mathrm{~S} \mathrm{~cm}^{-1}$, which is nearly negligible compared the total conductivity. Meanwhile, ultra-fine grained ones do not introduce extra electronic conduction. Therefore, it can be concluded that the improved electrical conductivity does not derive from electronic conduction.
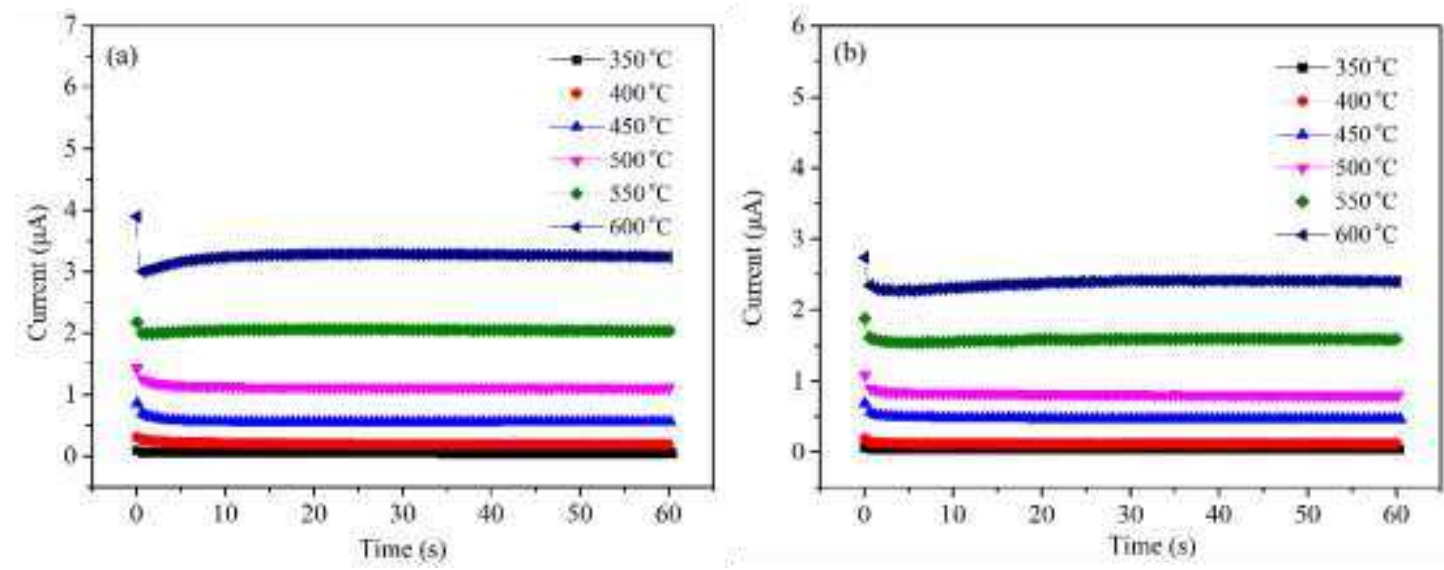

Fig. 5 I- $t$ curves of (a) micron (b) ultra-fine grained BiSDC-BCSBi electrolytes measured at $350-600{ }^{\circ} \mathrm{C}$ in air at a polarization potential of $0.8 \mathrm{~V}$.

Table 5 Electronic conductivities $\left(\sigma_{e}^{t}, \mathrm{~S} \mathrm{~cm}^{-1}\right)$ of micron and ultra-fine grained BiSDC-BCSBi electrolytes measured at $350-600{ }^{\circ} \mathrm{C}$.

\begin{tabular}{ccccccc}
\hline Sample & \multicolumn{7}{c}{ Measure temperate $\left({ }^{\circ} \mathrm{C}\right)$} \\
& 350 & 400 & 450 & 500 & 550 & 600 \\
\hline Micron BiSDC-BCSBi & 0.004 & 0.017 & 0.051 & 0.101 & 0.193 & 0.295 \\
Ultra-fine grained BiSDC-BCSBi & 0.004 & 0.010 & 0.043 & 0.073 & 0.145 & 0.220 \\
\hline
\end{tabular}

Oxygen and hydrogen concentration cells are designed to further clarify the ionic conduction behavior. The data shown in Fig.7 are the OCV, theoretical voltage $\left(E_{\text {Nernst }}\right)$ and ion transference number (OCV/E $\left./ E_{\text {Nernst }}\right)$ measured under dry oxygen, dry hydrogen and humid hydrogen. It can be found that in the dry oxygen concentration cell, the 
oxygen ion transference number of the ultra-fine grained electrolyte can reach $71.55 \%$, much higher than that of micron ones $(51.11 \%)$. In the hydrogen concentration cell, ultra-fine grained electrolytes showed slightly higher proton transference number. These results show that the increase of grain boundaries may promote both the transport of $\mathrm{O}^{2-}$ and $\mathrm{H}^{+}$, and mainly facilitate the transport of $\mathrm{O}^{2-}$.
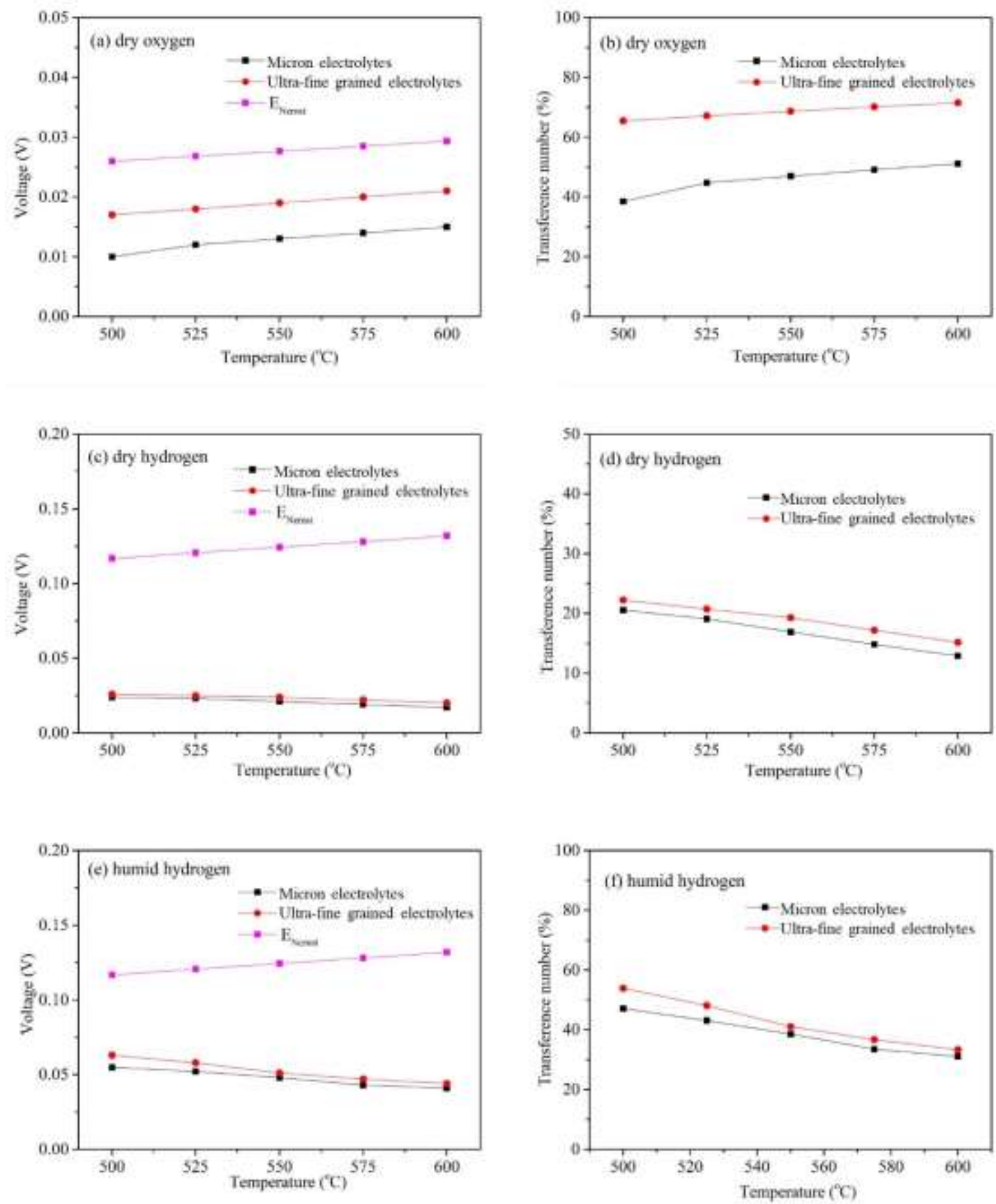

Fig. 6 (a, c, e) Voltage and (b, d, f) transference number of concentration difference cell of micron and ultra-fine grained BiSDC-BCSBi electrolytes at $500-600^{\circ} \mathrm{C}$. 
Fig. 7 shows the O 1s XPS spectrums of BiSDC-BCSBi electrolytes. The ultrafine grained electrolyte shows lower concentration of lattice oxygen (approximately at 528 $\mathrm{eV}[38])$ than the micron electrolyte, but higher concentration of $\mathrm{OH}$ groups and oxygen vacancies (approximately at $531 \mathrm{eV}$ [38]). The O-H group and oxygen vacancy concentration are semi-quantitatively analyzed by calculating the area of each peak position. The peak area of the $\mathrm{O}-\mathrm{H}$ group and oxygen vacancy is recorded as area 1 , and the lattice oxygen peak area is recorded as area 2, as shown in Table. 6. Comparing the area of the $\mathrm{O}-\mathrm{H}$ groups and oxygen vacancies (area 1) with the total peak area (area $1+$ area 2), the increase of grain boundaries is conducive to generate more $\mathrm{O}-\mathrm{H}$ groups and oxygen vacancies, thus enhancing the conduction of oxygen ions and protons.

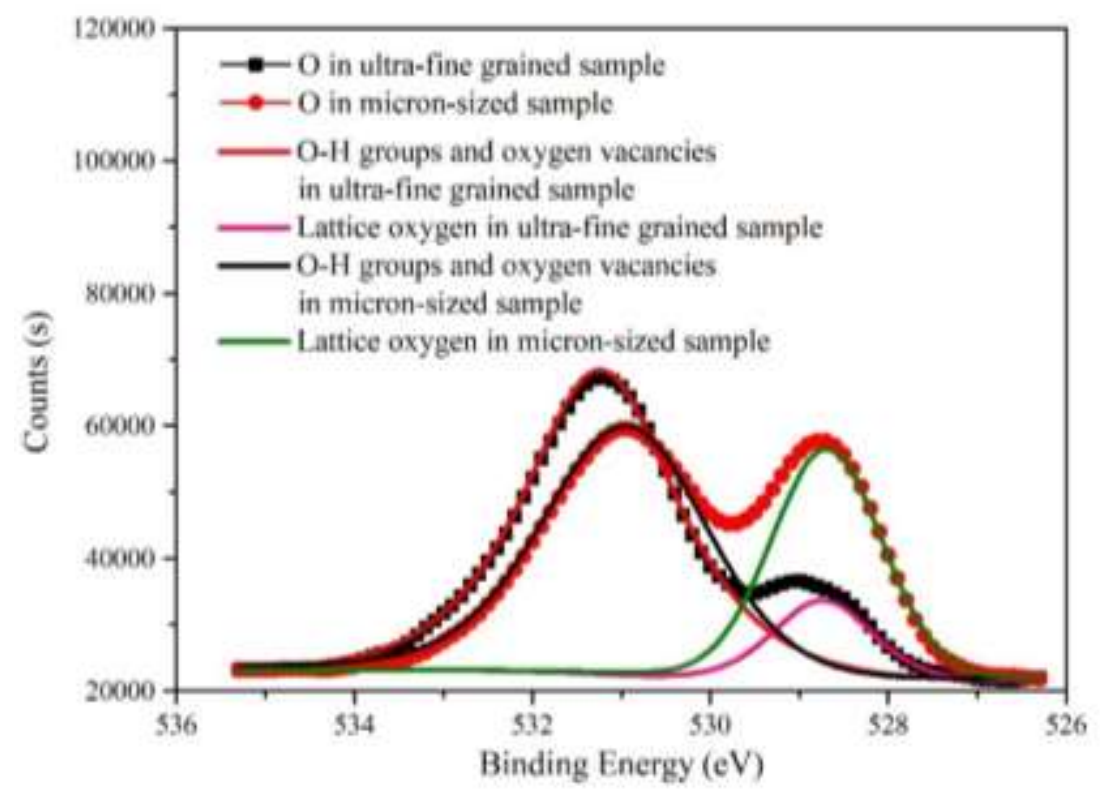

Fig. 7 O 1s XPS spectrums of micron and ultra-fine grained BiSDC-BCSBi electrolytes.

Table 6 Binding energy of fitting peak center and peak area and area ratio of fitting peak.

\begin{tabular}{cccccc}
\hline Sample & $\begin{array}{c}\text { Position1 } \\
(\mathrm{eV})\end{array}$ & $\begin{array}{c}\text { Position2 } \\
(\mathrm{eV})\end{array}$ & Area 1 & Area 2 & Area 1/ Area $(1+2)$ \\
\hline $\begin{array}{c}\text { Micron BiSDC-BCSBi } \\
\text { Ultra-fine grained }\end{array}$ & 530.94 & 528.69 & 90738.35 & 53226.71 & 0.630 \\
BiSDC-BCSBi & 531.24 & 528.73 & 149558.31 & 23657.53 & 0.863 \\
\hline
\end{tabular}

\subsection{Long-term stability}

In order to verify whether the increase of grain boundaries damage the stability of electrolytes, electrolyte-supported single cells were prepared to test the long-term stability using humid $\mathrm{H}_{2}$ as fuels and air as oxidants at $600{ }^{\circ} \mathrm{C}$, which is shown in Fig. 
8. Both ultrafine-grained and micron BiSDC-BCSBi electrolyte-supported cells maintain stable OCV values for testing of $100 \mathrm{~h}$. And ultrafine-grained ones show higher $\mathrm{OCV}$, due to their better electrical performance.

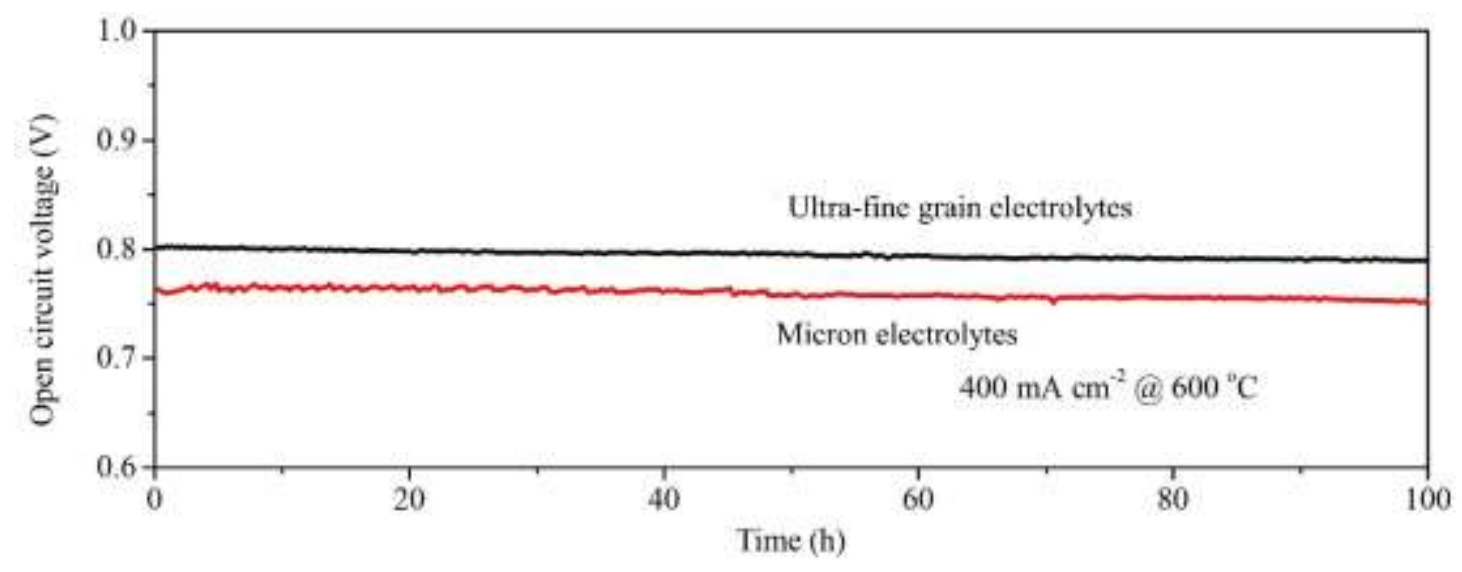

Fig. 8 The long-term stability of BiSDC-BCSBi electrolyte-supported single cells.

\section{Conclusion}

Ultra-fine grained $\mathrm{CeO}_{2} / \mathrm{BaCeO}_{3}$ based electrolytes were successfully fabricated by the TSS method, introducing more grain boundaries. Such electrolytes show much higher electrical conductivities than micron ones, due to in essence their decreased apparent unit grain boundary resistance. The beneficial grain boundary effect can ascribe two aspects including the decrease of space charge potential and the dilution of impurities. In the ultra-fine grained structure, there is no generation of additional electronic conduction. The good electrical performance comes from both enhanced transport of $\mathrm{O}^{2-}$ and $\mathrm{H}^{+}$caused by the relatively high concentration of $\mathrm{O}-\mathrm{H}$ groups and oxygen vacancies, during which the oxygen ion transference number is higher than the proton transference number. Finally, the electrolyte shows excellent long-term stability in SOFC operating conditions.

\section{Acknowledgements}

This work was supported by National Natural Science Foundation of China (Grant No. 51702189, 51802176), Natural Science Foundation of Shandong province (Grant No. ZR2020KE033, ZR2020ME05, ZR2019BEM013), Shandong Postdoctoral Innovation Foundation (Grant No. 201903069).

\section{Reference}


[1] Zhang Y, Knibbe R, Sunarso J, et al. Recent progress on advanced materials for solid-oxide fuel cells operating below $500{ }^{\circ} \mathrm{C}$. Adv Mater 2017, 29: 1700132.

[2] Li P, Yang W, Tian C, et al. Electrochemical performance of $\mathrm{La}_{2} \mathrm{NiO}_{4+\delta}$ $\mathrm{Ce}_{0.55} \mathrm{La}_{0.45} \mathrm{O}_{2-\delta}$ as a promising bifunctional oxygen electrode for reversible solid oxide cells. J Adv Ceram 2021, 10: 328-337.

[3] Danielle S, Yuval M, Mahdi H, et al. Defect chemistry of oxides for energy applications. Adv Mater 2018, 30: 1706300.

[4] Wei T, Qiu P, Yang J, et al. High-performance direct carbon dioxide-methane solid oxide fuel cell with a structure-engineered double-layer anode. J Power Sources 2021, 484: 229199.

[5] Zhu ZQ, Gong ZY, Qu P, et al. Additive manufacturing of thin electrolyte layers via inkjet printing of highly-stable ceramic inks. J Adv Ceram 2021, 10: 279-290.

[6] Oh EO, Whang CM, Lee YR, et al. Extremely thin bilayer electrolyte for solid oxide fuel cells (SOFCs) fabricated by chemical solution deposition (CSD). Adv Mater 2012, 24: $3373-3377$.

[7] Medvedev D, Murashkina A, Pikalova E, et al. $\mathrm{BaCeO}_{3}$ : Materials development, properties and application. Prog Mater Sci 2014, 60: 72-129.

[8] Wang Z, Huang X, Lv Z, et al. Preparation and performance of solid oxide fuel cells with YSZ/SDC bilayer electrolyte. Ceram Int 2015, 41: 4410-4415.

[9] Medvedev DA, Lyagaeva JG, Gorbova EV, et al. Advanced materials for SOFC application: strategies for the development of highly conductive and stable solid oxide proton electrolytes. Prog Mater Sci 2016, 75: 38-79.

[10] Sun W, Jiang Y, Wang Y, et al. A novel electronic current-blocked stable mixed ionic conductor for solid oxide fuel cells. J Power Sources 2011, 196: 62-68.

[11] Ding H, Guo X, Li Jiao, et al. Processing and characterization of novel $\mathrm{Ce}_{0.8} \mathrm{Sm}_{0.1} \mathrm{Bi}_{0.1} \mathrm{O}_{2-\delta}{ }^{-} \mathrm{BaCe}_{0.8} \mathrm{Sm}_{0.1} \mathrm{Bi}_{0.1} \mathrm{O}_{3-\delta}$ ( $\left.\mathrm{BiSDC}-\mathrm{BCSBi}\right)$ composite electrolytes for intermediate-temperature solid oxide fuel cells. Int J Hydrogen Energ 2020, 46: 75157527.

[12] Li B, Liu S, Liu X, et al. Electrical properties of SDC-BCY composite electrolytes for intermediate temperature solid oxide fuel cell. Int J Hydrogen Energ 2014, 39: 
14376-14380.

[13] Kumar VP, Reddy YS, Kistaiah P, et al. Thermal and electrical properties of rareearth co-doped ceria ceramics. Mater Chem Phys 2008, 112: 711-718.

[14] Li ZP, Mori T, Ye F, et al. Dislocation associated incubational domain formation in lightly gadolinium-doped ceria. Microsc. Microanal 2010,17: 49-53.

[15] Wandekar RV, Wani BN, Bharadwaj SR. Disparity in properties of 20-mol\% Eudoped ceria synthesized by different routes. Solid State Sci 2010, 12: 8-14.

[16] Jamnik J, Pejovnik S, Maier J. Interfaces in solid ionic conductors: Equilibrium and small signal picture. Solid State Ionics 1995, 75: 51-58.

[17] Yan D, Liu X, Liu Q, et al. Study on rare earth electrolyte of SDC. J Rare Earth 2010, 028: 914-916.

[18] Badwal S, Drennan J. Yttria-zirconia. Effect of microstructure on conductivity. $J$ Mater Sci 1987, 22: 3231-3239.

[19] Tschpe A, Sommer E, Birringer R. Grain size-dependent electrical conductivity of polycrystalline cerium oxide. Solid State Ionics 2001, 139: 255-265.

[20] Christie GM, Berkel FPF. Microstructure-ionic conductivity relationships in ceriagadolinia electrolytes. Solid State Ionics 1996, 83: 17-27.

[21] Yan D, Liu X, Bai X, et al. Electrical properties of grain boundaries and size effects in samarium-doped ceria. J Power Sources 2010, 195: 6486-6490.

[22] Xin G, Waser R. Electrical properties of the grain boundaries of oxygen ion conductors: Acceptor-doped zirconia and ceria. Prog in Mater Sci 2006, 51: 151-210. [23] Anselmi TU, Maglia F, Chiodelli G, et al. Nanoscale effects on the ionic conductivity of highly doped bulk nanometric cerium oxide. Adv Funct Mater 2006, 16: 2363-2368.

[24] Bellino MG, Lamas DG, Walsöe NE. A mechanism for the fast ionic transport in nanostructured oxide-ion solid electrolytes. Adv. Funct. Mater 2006, 18: 3005-3009. [25]Enrique RT, Jaime SS, Ruben VM, et al. Microstructure and electrical transport in nano-grain sized $\mathrm{Ce}_{0.9} \mathrm{Gd}_{0.1} \mathrm{O}_{2-\delta}$ ceramics. J Solid State Chem 2007, 180: 3093-3100. [26] An J, Bae J, Hong S, et al. Grain boundary blocking of ionic conductivity in nanocrystalline yttria-doped ceria thin films. Scripta Mater 2015, 104: 45-48. 
[27] Iguchi F, Sata N, Yugami H. Proton transport properties at the grain boundary of barium zirconate based proton conductors for intermediate temperature operating SOFC. J Mater Chem 2010, 20: 62-65.

[28] Pergolesi D, Fabbri E, Epifanio D, et al. High proton conduction in grainboundary-free yttrium-doped barium zirconate films grown by pulsed laser deposition. Nat Mater 2010, 9: 846-852.

[29] Aarthi U, Babu KS. Grain boundary space charge modulation in $\mathrm{BaZr}_{0.8} \mathrm{Y}_{0.2-}$ ${ }_{\mathrm{x}} \mathrm{M}_{\mathrm{x}} \mathrm{O}_{3 \delta}$ with transition metal $(\mathrm{M}=\mathrm{Ni}, \mathrm{Co}, \mathrm{Fe}$, and $\mathrm{Zn})$ co-doping. Int J Hydrogen Energ 2020, 45: 29356-29366.

[30] Shimonosono T, Hirata Y, Ehira Y, et al. Electronic conductivity measurement of Sm- and La-doped ceria ceramics by Hebb-Wagner method. Solid State Ionics 2004, 174: $27-33$.

[31] Sun HB, Rainwater BH, Xiong X, et al. Interfacial effects on electrical conductivity in ultrafine-grained $\mathrm{Sm}_{0.2} \mathrm{Ce}_{0.8} \mathrm{O}_{2-\delta}$ electrolytes fabricated by a two-step sintering process. Int J Hydrogen Energ 2017, 42: 11823-11829.

[32] Irvine JTS, Sinclair DC, West AR. Electroceramics: characterization by impedance spectroscopy. Adv Mater 1990, 21: 132-138.

[33] Pandey R, Singh P. Electrical conductivity of YSZ-SDC composite solid electrolyte synthesized via glycine-nitrate method. Ceram Int 2017, 43: 11692-11698. [34] Nielsen J, Hjelm J. Impedance of SOFC electrodes: A review and a comprehensive case study on the impedance of LSM: YSZ cathodes. Electrochimica Acta 2014, 115: $31-45$.

[35] Deng H, Zhang W, Wang X, et al. An ionic conductor $\mathrm{Ce}_{0.8} \mathrm{Sm}_{0.2} \mathrm{O}_{2-\delta}$ (SDC) and semiconductor $\mathrm{Sm}_{0.5} \mathrm{Sr}_{0.5} \mathrm{CoO}_{3}$ (SSC) composite for high performance electrolyte-free fuel cell. Int J Hydrogen Energ 2017, 42: 22228-22234.

[36] Xin G, Zhang Z. Grain size dependent grain boundary defect structure: case of doped zirconia. Acta Mater 2003, 51: 2539-2547.

[37] Chen G, Zhang X, Luo Y, et al. Ionic conduction mechanism of a nanostructured BCY electrolyte for low-temperature SOFC. Int J Hydrogen Energ 2019, 45: 2410824115. 